Stéphane Pesnel | Université Paris-Sorbonne, stephanepesnel.sorbonne@gmail.com

\title{
Spurenlesen in den Bergen \\ Die Natur als Palimpsest des Krieges in Mario Rigoni Sterns Erzählwerk
}

Ich bin kein Historiker, der in alten Handschriften oder in Archivdokumenten forscht. Ich begnüge mich damit, ein Erzähler zu sein, der einige Spuren befolgt, die ihm seine heimatliche Gegend bietet. ${ }^{1}$

Das Werk des italienischen, aus Asiago (im Nordwesten der Region Venetien, Provinz von Vicenza) stammenden Schriftstellers Mario Rigoni Stern (1921-2008) setzt sich bekanntlich aus zwei Hauptkomplexen zusammen, die sich eher thematisch als formal bestimmen lassen: Einerseits gibt es die Naturgeschichten, deren Titel meistens auf die Flora, die Fauna, die Jahreszeiten oder auf persönliche Erlebnisse mit der Natur verweisen, andererseits die Kriegsgeschichten, die entweder individuelle Erlebnisse des Verfassers oder auch, von exemplarischen Schicksalen ausgehend, kollektive Erlebnisse seiner norditali-

1 Rigoni Stern: Segni lontani, S. 83: »Non sono uno storico che ricerca nei manoscritti antichi o nei documenti degli archivi; mi accontento di essere un narratore che segue qualche traccia della sua terra.« Da der vorliegende Beitrag ausschließlich auf Erzählungen Rigoni Sterns Bezug nimmt, wird in den Quellenangaben der Name des Autors von nun an systematisch ausgespart. Die Übersetzungen der Zitate stammen vom Verfasser des Beitrags.
Zu den Grundkonstanten von Mario Rigoni Sterns Erzählungen zum Ersten Weltkrieg gehört die Dialektik von Natur und Geschichte. Meistens bildet die Natur ihrer Erhabenheit wegen einen radikalen Gegenpol zum Handeln der Menschen. Zuweilen kommt es aber zu einer Kontamination der bisher unberührten Landschaften durch die Gewalt des Krieges. In einigen sracconti di guerra wird die Natur zu einem regelrechten Palimpsest. Der Erzähler unternimmt es dann, die in den Landschaften hinterlassenen historischen Spuren zu entziffern, um das Kriegsgeschehen und das Leben einfacher Soldaten zu rekonstruieren. Natur, Krieg, Geschichte und Gedächtnis werden subtil miteinander in Verbindung gebracht. 
enischen Mitbürger dichterisch verarbeiten. Eigentlich ist diese Zweiteilung recht vereinfachend, da es bei Rigoni Stern öfters zu einer Überlagerung der beiden Themenbereiche innerhalb einer bestimmten Erzählung kommt. Kriegsgeschichten spielen sich meistens vor einer landschaftlichen Folie ab, deren Beschreibung nicht ausgespart wird, Naturgeschichten lassen oft Spuren des Krieges an den Tag treten.

Begonnen hat die literarische Karriere Mario Rigoni Sterns mit einem fulminanten Bucherfolg: 1953 veröffentlicht der Schriftsteller und Herausgeber Elio Vittorini, Autor des berühmten Buches Conversazione in Sicilia (Gespräch in Sizilien), Rigoni Sterns Erstling, Il sergente nella neve (Alpini im russischen Schnee) in der Reihe >I Gettoni « im namhaften Verlag Einaudi (Turin). ${ }^{2}$ Es handelt sich dabei um ein Erzählwerk mit stark autobiographischem Hintergrund, ${ }^{3}$ dessen Entstehung auf eine innere Notwendigkeit zurückzuführen ist. Der Autor, der im Zweiten Weltkrieg an zahlreichen Manövern, Märschen und Gefechten in Albanien und in Russland teilgenommen hat, bevor er im September 1943 von den Deutschen gefangen genommen und nach Ostpreußen in ein Kriegsgefangenenlager geschickt wurde, ${ }^{4}$ hat nämlich fast durchgehend Tagebuch geführt (wenn auch öfters minimalistisch, sich auf kurze Einträge beschränkend), bis auf die Periode, wo er sich auf dem Rückmarsch aus Russland befand. ${ }^{5}$ Mit Il sergente nella neve ging es ihm darum, eine Lücke zu schließen ${ }^{6}$ und die eigenen Erlebnisse, die jenen ‘zeitenthobenen Tagen entsprachen, im Nachhinein literarisch festzuhalten. ${ }^{7}$ Viele andere Erzählungen Rigoni Sterns kreisen um seine russische Periode oder

2 Von Mario Rigoni Stern ist erstaunlich wenig ins Deutsche übertragen worden (er ist in Frankreich etwas bekannter). Offensichtlich sind bis jetzt nur vier Werke in deutscher Übersetzung erschienen: Alpini im russischen Schnee [Il sergente nella neve]; Füchse unter Sternen [Il bosco degli urogalli]; Tönle [Storia di Tönle]; Geblendet und betrogen [Lultima partita a carte].

3 Der Untertitel lautet ja »Erinnerungen vom Rückzug aus Russland « (»Ricordi della ritirata di Russia«). Bei Mario Rigoni Stern sind die tradierten Gattungsbezeichnungen der Epik aufgrund der Nähe seiner Erzählprosa zum Autobiographischen (und zuweilen sogar zum Dokumentarischen) problematisch, wenn nicht irrelevant. Wohl aufgrund seiner Vorliebe für Naturdarstellungen und -geschichten bezeichnet er sich selbst als Vertreter einer "ländlichen Epik« (Rigoni Stern/Milani: Storia di Mario, S. 74: »un'epica paesana«).

4 Siehe dazu u.a. die autobiographische Erzählung Ritorno nel Lager I/B, S. 67-78.

5 In der Erzählung Quasi una tregua aus der Sammlung Aspettando l'alba nennt er diese Tage im Winter 1943 »die Tage ohne Datum «(S. 55: »[i giorni] senza data «). Der Eindruck eines Fehlens zeitlicher Anhaltspunkte wird durch die Beschaffenheit der durchwanderten Landschaften noch verschärft: »Wir marschierten und marschierten und hatten den Eindruck, immer noch am selben Ort zu sein, weil die Steppe wie das Meer ist." (Fantasie di Ferragosto, S. 234: „Si camminava e si camminava e sembrava di essere sempre nello stesso posto perché la steppa è come il mare.«)

6 Siehe dazu: Rigoni Stern/Milani: Storia di Mario, S. 9.

7 Die erste Fassung dieses Erinnerungswerkes geht schon auf die Zeit der Gefangenschaft in Ostpreußen zurück. 
um die Zeit im Kriegsgefangenenlager - sowie um die Reise nach Russland, die er fast drei Jahrzehnte nach Ende des Zweiten Weltkriegs unternimmt, um die damals durchwanderten Orte und Landschaften wiederzusehen. ${ }^{8}$

Einige Jahre nach seinem literarischen Debüt hat Mario Rigoni Stern die historische Spannweite seines erzählerischen Schreibens erweitert und begonnen, sich dem Ersten Weltkrieg zuzuwenden. Seine Heimat, die Hochebene von Asiago, sowie relativ nahe gelegene Regionen - die Carnia im Friaul, die Isonzo-Gegend (die heutige Grenzregion zwischen Slowenien und Italien) - sind nämlich im Ersten Weltkrieg der Schauplatz von zahlreichen Kämpfen zwischen der österreichisch-ungarischen Armee und den italienischen Streitkräften gewesen. ${ }^{9}$ Selbst wenn Mario Rigoni Stern den `Großen Krieg` nicht selber erlebt hat (ist er doch erst 1921 geboren), ist er schon früh mit diesem Großereignis sowie mit Zeugnissen, Erinnerungen und Gesprächen, die den Krieg zum Inhalt hatten, vertraut geworden. Als Kind hat er die noch frischen Spuren der Gefechte bei Spaziergängen mit seinem Vater beobachten können; darüber berichtet er in einer Erzählung mit dem Titel Malghe (Almen). ${ }^{10}$ In einer anderen, Amore e morte nel bosco incantato (Liebe und Tod im verzauberten Wald) betitelten Erzählung stellt er aber fest, dass eben diese Spuren jetzt, im ausgehenden 20. Jahrhundert, zu verschwinden drohen, dass sie von dem Verfließen der Zeit und der Überwucherung der Vegetation allmählich verwischt werden. Sie seien nur noch für denjenigen sichtbar, der richtig zu schauen wisse:

In der unmittelbaren Nachkriegszeit befanden sich [die] Friedhöfe [der Soldaten] in diesen kleinen Tälern, und das Gelände war von kriegerischen Überbleibseln durchsetzt. Nun hat der Wald alles verdeckt, aber für denjenigen, der richtig zu schauen weiß, sind die Spuren der Schützengräben und der Unterschlüpfe noch durchaus sichtbar. Wer weiß, wie viele Dinge zwischen den Wurzeln der Bäume verborgen liegen. Die Bäume erzählen dem Himmel von den Geheimnissen der Erde. ${ }^{11}$

Die Landschaft mit scharfem Blick, mit wissendem Auge lesen, das ist eines der Grundmotive in Rigoni Sterns Erzählwerk. Der Erzähler, der mit den

8 Das paradigmatische Werk dafür ist Ritorno sul Don (1973), das man als Pendant zu Il sergente nella neve (1953) ansehen darf.

9 Siehe dazu: Labanca/Überegger (Hgg.): La Grande Guerra italo-austriaca 1914-1918.

10 Malghe, S. 229-232, siehe insbesondere S. 230. In dieser kurzen Erzählung entfaltet der Autor die Auffassung vom Spaziergang als einer Schule des Sehens, und in der unmittelbar darauffolgenden (Fantasie di ferragosto) ein damit eng verbundenes Thema: das Schreiben (und das Spazierengehen) als Reise ins (oder eher: durchs) eigene Gedächtnis (ebd., S. 233: "un viaggio nella memoria «).

11 Amore e morte nel bosco incantato, S. 228: »In quel dopoguerra, per queste vallette c'erano i loro cimiteri e il terreno era cosparso di residuati bellici. Ora il bosco ha ricoperto tutto, ma per chi sa guardare ancora sono ben visibili gli scavi delle trincee e dei ricoveri. Tra le radici degli alberi chissà quante cose sono nascoste. Gli alberi raccontano al cielo i segreti della terra.« 
Berglandschaften im Vicentino und mit der italienischen Geschichte des 20. Jahrhunderts vertraut ist, führt uns auf seinen Spaziergängen und Wanderungen mit und zeigt uns das, was wir mit unserem ungenau schauenden Auge nicht zu erblicken vermögen. Dass es sich bei dem Motiv der Überwucherung durch die Vegetation z.T. um eine literarische Stilisierung (im vorliegenden Fall: um eine Metapher des Vergessens) und um eine schriftstellerische Übertreibung handelt, leuchtet wohl ein, sind doch die Spuren und Narben des Ersten Weltkrieges in den norditalienischen Landschaften noch an vielen Stellen zu erblicken, genauso wie sie die nord- und ostfranzösischen Landschaften nach einem Jahrhundert immer noch prägen. ${ }^{12}$

Mario Rigoni Sterns Erzählungen zum Zweiten Weltkrieg und diejenigen zum Ersten Weltkrieg sind von demselben Willen getragen, ein Bollwerk gegen das Vergessen zu errichten, Zeugnis abzulegen, ${ }^{13}$ wobei sich der Schriftsteller im Falle des Ersten Weltkriegs nicht auf persönliche Erlebnisse und Erinnerungen stützen kann, sondern auf die Lektüre von allerlei Dokumenten, Memoiren und Geschichtsbüchern angewiesen ist. In seinen Erzählungen, in die zuweilen wissenschaftliche Quellen bzw. Bezüge auf Werke von Historikern eingeflochten sind, erscheint uns Rigoni Stern als ein leidenschaftlicher Leser von historischen Studien zum Ersten Weltkrieg. ${ }^{14}$ Der Rückgriff auf solches Quellenmaterial kompensiert das Fehlen persönlicher Erlebnisse und Erinnerungen, das sich im vorliegenden Fall nicht als besonders gravierend erweist, da ihm die eigene Kriegserfahrung aus den 1940er Jahren seiner Meinung nach ein epochenübergreifendes Einfühlungsvermögen verleiht. Als Soldat im Zweiten Weltkrieg sind ihm, so lässt er zuweilen durchblicken, ähnliche existentielle Grunderfahrungen wie seinen Leidensgenossen im Ersten Weltkrieg zuteil geworden, die mit elementaren Situationen wie Hunger, Erschöpfung, Einsamkeit, Angst, Todesnähe, aber auch Solidarität, Mitgefühl, Freundschaft zu tun haben. Durch die eben angesprochene Mischung aus historischem Wissen und humaner Empathie verschafft sich der Verfasser einen eigenen Zugang

12 So behauptet der Historiker Marco Mondini: »Selten hat ein Krieg so tiefe Spuren in der Landschaft hinterlassen wie der Erste Weltkrieg in Italien. Die Spuren der in den Jahren 1915-1918 gelieferten Schlachten sind überall sichtbar." (Mondini: Andare per i luoghi della Grande Guerra, S. 7: »Raramente una guerra ha lasciato segni così profondi sul paesaggio come il primo conflitto mondiale in Italia. Le traccie delle battaglie combattute tra 1915 e 1918 sono visibili ovunque.")

13 Siehe z.B.: Così a diciottanni andammo in guerra, S. 41.

14 Siehe insbesondere die in der ersten Sektion des Bandes I racconti di guerra zitierten Quellen und Studien, die von Rigoni Sterns intensiver historischer Beschäftigung mit dem Ersten Weltkrieg zeugen. 
zum Ersten Weltkrieg, dessen Authentizität und Tiefe trotz der Tatsache des Nachgeborenseins nicht in Frage zu stellen sind.

Was Mario Rigoni Sterns Lektüren angeht, ist eines anzumerken: Der Autor zitiert mit Vorliebe aus Primärquellen (Briefen, Berichten, Zeugnissen) oder aus historischen Studien, welche sich mit dem Leben einfacher Soldaten an der Front oder der Zivilbevölkerung befassen. Er distanziert sich dabei von der globalisierenden Sicht jener Historiker, die sozusagen einen Blick von oben auf den Verlauf des Krieges werfen. Mit der Wahl eines niedrigeren und bescheideneren Standpunktes gelingt es den von Rigoni Stern zitierten (Lokal)historikern, das Schicksal unbekannter, von der akademischen Geschichtsschreibung vernachlässigter Menschen dem Vergessen zu entreißen - und eben dies steht im Einklang mit seiner eigenen Poetik. Im ersten, dem >Großen Krieg` gewidmeten Teil der Sammlung I racconti di guerra (Kriegserzählungen), die vom Autor selbst zusammengestellt wurde,${ }^{15}$ wird das Los von einfachen Infanteristen geschildert, von Soldaten aus dem Trentino und aus den Julischen Alpen, die in den Reihen der österreichisch-ungarischen Armee an der Ostfront (in Galizien oder in der Bukowina) eingesetzt wurden, von Soldaten, die in russische Kriegsgefangenschaft gerieten. Es geht auch um die Gewissensfragen der Gorizianer und der Triestiner, die nicht wussten, ob sie in die österreichisch-ungarische oder in die italienische Armee gehörten. Und es geht um die Einwohner des Altipiano dei Sette Comuni (Hochebene der Sieben Gemeinden, Mario Rigoni Sterns Heimat), die sich auf die Flucht begeben ${ }^{16}$ - oder in erbärmlichen Verhältnissen leben mussten, wenn sie sich dazu entschlossen hatten, die Flucht eben nicht zu ergreifen. Die beiden Flüchtlingswellen vom Jahre 1916 und vom Jahre 1917 (nach der Niederlage bei Caporetto/ Karfreit/ Kobarid) sind laut Rigoni Stern von den Historikern unzureichend behandelt worden und so soll sein erzählerisches Werk einen Beitrag zur Reevaluierung von einigen vernachlässigten historischen Aspekten leisten.

Darüber hinaus geht der Wille, unbekannt-unscheinbare Schicksale zu rehabilitieren und dem Vergessen zu entreißen, unmissverständlich in die Richtung einer anders gedachten Geschichtsschreibung, wie sie Walter Benjamin in seinen Thesen zum Begriff der Geschichte entworfen hat, und die nicht mehr die Sieger in den Mittelpunkt des Interesses rücken soll, sondern eher die Besiegten, die Namenlosen, die Unscheinbaren. Literarisches

$16 \mathrm{Zu}$ den Flüchtlingswellen aus Norditalien und insbesondere aus dem 1916 zerbombten Asiago siehe z.B.: Il naufragio dei contadini, S. 48-52. Zum Wiederaufbau der Hochebene von Asiago siehe: La ricostruzione dell'Altipiano di Asiago, S. 103-141. 
Schreiben hat bei Mario Rigoni Stern stets mit moralischer Verantwortung zu tun, es ist in seinen Augen die Pflicht des Schriftstellers, denjenigen eine Stimme zu geben, die nicht sprechen können bzw. konnten (»dare la voce a chi non poteva più parlare «). ${ }^{17}$ Diese Haltung ist nicht nur als eine Hommage an die ältere Generation zu verstehen, sondern auch als eine Hinwendung zur jüngeren Generation: In einem sowohl vergangenheitsbezogenen als auch zukunftsgerichteten Erzählgestus möchte Mario Rigoni Stern Zeugnis ablegen. ${ }^{18}$ Dabei gebärdet er sich nie schulmeisterlich, belehrend, sondern sieht seine Aufgabe darin, einfach und schlicht, ohne Glossen das zu sagen, was gewesen ist - ein historisches und menschliches Wissen zu vermitteln, damit es nicht aufgrund der Gleichgültigkeit und Vergesslichkeit unseres Zeitalters verlorengeht. Der eigenen Generation wirft er vor, diejenige der Väter nicht genug nach dem genauen Verlauf und der konkreten Wirklichkeit des Ersten Weltkriegs gefragt zu haben. ${ }^{19}$ Diesem Fehler soll durch das Erzählen abgeholfen werden. ${ }^{20}$

Im Erzählwerk Mario Rigoni Sterns wird der Erste Weltkrieg oftmals als eine Zäsur sondergleichen bezeichnet. Auf seine heimatliche Region bezogen heißt es, dass der Krieg ganze Dörfer und Landschaften verwüstet, eine ganze Zivilisation zerstört hat: »Das materielle Gedächtnis meiner Heimat in den Bergen ist vom Großen Krieg zerstört worden. Es wurde von hunderttausenden Bomben und Granaten zerstückelt. Nun liegt es verborgen unter den Straßen, unter den Einzelhäusern, unter den Wohngebäuden und unter den Parkplätzen. ${ }^{21}$ So die Erzählung Cartoline (Postkarten). Und in der Erzählung Segni lontani (Entfernte Zeichen) äußert der Schriftsteller Folgendes: »Die Spuren, die Bräuche und auch die Sprache

17 In diesem Motto erblickt Folco Portinari das (ethische) Grundprinzip von Mario Rigoni Sterns Erzählwerk. Siehe seine Einleitung zu I racconti di guerra, S. V-XXIII. Ein anderes Motto, unter dem man ganze Teile seines Werkes zusammenfassen könnte, ist der Vers von Primo Levi, den er in der Erzählung Ritorno nel Lager I/B zitiert: »Meditiert darüber, dass dies gewesen ist.« (S. 71: "Meditate che questo è stato.«)

18 An seinen Schriftstellerkollegen Emilio Lussu und Carlo Emilio Gadda lobt Mario Rigoni Stern die Tatsache, dass sie »Erzähler-Zeugen « seien (Musil in trincea, S. 41: "narratori testimoni«). Eine doppelte Eigenschaft, die zweifelsohne auch ihn selbst charakterisiert. Rigoni Stern bezieht sich mehrfach auf Lussus autobiographisches Zeugnis Un anno sull'Altipiano (1938), in dem der aus Sardinien stammende Schriftsteller über seine Teilnahme am Gebirgskrieg auf dem Altipiano di Asiago berichtet.

19 Il Piave mormorava, S. 23.

20 Zur Bewältigung des Ersten Weltkriegs in der italienischen Gesellschaft siehe u.a. Isnenghi: Il mito della Grande Guerra sowie Labanca (Hg.): Dizionario storico della Prima Guerra mondiale.

21 Cartoline, S. 78: "La memoria materiale del mio paese montanaro è stata distrutta dalla Grande Guerra. Ė stata frantumata da centinaia di migliaia di bombe e granate. Rimane nascosta sotto le strade, sotto le case, sotto i condominî e i parcheggi.« 
hat der Erste Weltkrieg zerstört, indem er unsere Gegenden von Grund auf erschüttert und alle ihre Einwohner als Flüchtlinge und als Verzweifelte in die verschiedensten Regionen Italiens vertrieben hat. ${ }^{22}$ Neue Dörfer und Städte seien auf dem Schutt des Ersten Weltkriegs entstanden, unter der Oberfläche liege also eine Welt begraben, deren Überreste manchmal bei Bauarbeiten wieder an den Tag kämen. Rigoni Stern erwähnt, wie fasziniert er ist, wenn er auf einer Baustelle Fragmente der Vergangenheit, Scherben der Geschichte wieder hervortauchen sieht: »Es sind dies die Dinge, die mir die Tragödie meines Volkes erzählen, und die mich zutiefst rühren.«³

In seinem Werk ist oft von Spuren der Geschichte die Rede, wobei diese Spuren meistens die Form von alltäglichen Gegenständen annehmen. ${ }^{24}$ Sie bilden das bescheidene, schlichte, konkrete Material, anhand dessen der zum Sehen und zum Interpretieren befähigte Erzähler Vergangenheit rekonstruieren, individuelles und kollektives Schicksal miteinander verknüpfen, Lokalgeschichte in den größeren Rahmen der Weltgeschichte einordnen kann. Eine der schönsten Erzählungen ist in dieser Hinsicht diejenige mit dem Titel La bottiglia ritrovata (Die wiedergefundene Flasche) in der Sammlung Aspettando l'alba (Beim Warten auf die Morgendämmerung). Es geht in dieser Erzählung um eine Flasche Grappa, die viele Jahre nach dem Ersten Weltkrieg im Gebirge wieder aufgefunden und deren Weg bis in die Berge, die damals Kriegsschauplatz waren, nachgezeichnet wird. Aus einem Kurzurlaub hatte ein italienischer Offizier diese Flasche für einen seiner Vorgesetzten mitgebracht und sie behutsam unter Steinen versteckt; als sein Bataillon plötzlich abziehen musste, hatte er beim eiligen Packen vollkommen vergessen, sie wieder in seinen Rucksack zu stecken. Diese Flasche wird nicht nur zum literarischen Vorwand, um die Wege und Irrwege der italienischen Truppen durch die Berglandschaften im Kontext des Vormarsches der österreichischen Armee zu erzählen, sondern auch

22 Segni lontani, S. 87: "A cancellare tracce, costumi e anche lingua è stata la Prima Guerra mondiale che sconvolse le nostre contrade e disperse per l'Italia, profughi e disperati, tutti gli abitanti residenti."

23 Cartoline, S. 78: "Sono queste le cose che mi raccontano la tragedia della mia gente, e che mi danno emozione."

24 So z.B. die (Feld)Postkarten, von denen in der Erzählung Quasi una tregua die Rede ist: "Ich lese portofreie Postkarten, auf denen man heroische Bilder sehen, Mussolinische oder D’Annunzianische Motti lesen kann; von den Schriftzügen, von der Kürze des Geschriebenen, von dem Datum ausgehend kann ich eine spannungsgeladene Situation rekonstruieren; von der Länge des Geschriebenen, einen ruhigen Augenblick. (Quasi una tregua, S. 54: »Leggo cartoline in franchigia che hanno illustrazioni eroiche e frasi mussoliniane o dannunziane; dalla calligrafia e dalla brevità dello scritto, dalla data, ricostruisco una situazione drammatica; dalla lunghezza un momento tranquillo.«) 
zu einem regelrechten Träger der Erinnerung an diese Schicksale, die die Grenzgebiete zwischen Italien und Österreich als Folie hatten.

Geschichte rekonstruiert Mario Rigoni Stern übrigens nicht nur anhand von Gegenständen, sondern auch anhand von charakteristischen Orten, die er historisch kontextualisiert. Beispielhaft für diese an der Topographie und an Raumstrukturen orientierte literarische Geschichtsschreibung ist die Erzählung Osteria di confine (Grenzschenke), in der die österreichischitalienische Geschichte vom 18. bis zum 20. Jahrhundert (einschließlich des Ersten Weltkrieges) umrissen wird. ${ }^{25}$ Dabei bevorzugt Rigoni Stern wiederum nicht den globalen, sondern den lokalen, realitätsnahen Blick.

Unscheinbare und doch höchst signifikante Spuren der Geschichte, insbesondere des Großen Krieges, gibt es viele in Mario Rigoni Sterns Erzählwerk: die schon erwähnte Flasche Grappa, eine Metallscherbe von einer Glocke, ${ }^{26}$ Postkarten, ${ }^{27}$ eine Nussholzschachtel mit alten Briefen, ${ }^{28}$ die Metallspitze von einem Bergstock, ${ }^{29}$ und vor allem Steine, die in den Bergen verstreut sind und u.a. an die Irredentisten erinnern, ${ }^{30}$ die nicht in den Reihen der österreichisch-ungarischen Armee hatten kämpfen wollen, oder auch an die Opfer des Ersten Weltkrieges. ${ }^{31}$ Diese Spuren sind historische Indizien, mittels derer der Erzähler Vergangenheit und Geschichte rekonstruieren, dem Vergessen entreißen kann. Man könnte hier von einer `objektalen`, gegenstandsbezogenen Geschichtsschreibung sprechen..$^{32}$ Dieser sowohl ästhetische als auch methodologische Ansatz des geschichtsschreibenden Dichters nimmt erstaunlicherweise neuere Tendenzen der Historiographie und der Museumsgestaltung vorweg, wie sie in der beeindruckenden Ausstellung zum Ersten Weltkrieg im Deutschen Historischen Museum zu Berlin (2014) zu beobachten gewesen

Osteria di confine, S. 52-63.

Scampanio profano, S. 144.

Cartoline, S. 78-82; Quasi una tregua, S. 54.

Quasi una tregua, S. 51-66.

I miei sentieri sotto la neve, S. 124.

»Hier und da in meinen Bergen des Altipiano erinnern viele Steine an die jungen >Irredentisten denen der Tod im Kampf lieber war als die Gefangenschaft, da man sie schließlich als Verräter und Deserteure erschossen hätte." (Miserere nel vento dei Carpazi, S. 11: "Tra le mie montagne dell'Altipiano molti lapidi restano a ricordare i giovani irredentisti< che preferivano morire in combattimento piuttosto che cadere prigionieri, in quanto la fucilazione come traditori e disertori sarebbe stata la loro fine.«)

Amore e morte nel bosco incantato, S. 228.

In der Erzählung Cartoline bezeichnet er diese unscheinbaren Dinge als »kleine Gegenstände, in denen sich das historische Gedächtnis kristallisiert « (Cartoline, S. 78: "piccoli oggetti in cui si coagula la memoria storica «). 
sind. ${ }^{33}$ Bei allem unleugbaren Realitätsgehalt können diese gegenständlichen Spuren zugleich als Metaphern für das eigene erzählerische Schreiben, für die eigene Erzählpoetik verstanden werden: Die Nussholzschachtel und die Postkarten versinnbildlichen und materialisieren gleichzeitig das eigene, innere Archiv der Erinnerungen, ${ }^{34}$ und die überall in den Bergen verstreuten Gedenksteine stehen metaphorisch, wenn nicht metapoetisch, für die eigenen Kurzgeschichten und Erzählungen, die keinen Anspruch auf eine globalisierende und synthetisierende Geschichtsschreibung erheben, sondern sich eher als bescheidene Erinnerungssplitter ausnehmen, dank denen unbekannte Schicksale, insbesondere von Einwohnern des Altipiano di Asiago und von Soldaten, die es dorthin verschlagen hat, nicht in Vergessenheit geraten mögen. Auch Grabsteine auf dem Dorffriedhof, Auflistungen von Namen, hier und dort verstreute Inschriften fungieren als Auslöser des Erinnerungs- und Vergegenwärtigungsprozesses: »In jenen Namen, in jenen Daten erkenne ich vieles, was zu meinem eigenen Leben, zum Leben der Gegend gehört, die große und die kleine Geschichte. Die Griechen hatten recht, wenn sie sagten, die Musen seien die Töchter des Gedächtnisses. « ${ }^{35}$

Abgesehen von den vereinzelten Zeichen und Spuren, die auf vergangene Jahrzehnte und insbesondere auf den Ersten Weltkrieg hinweisen, könnte man sagen, dass die Alpenlandschaft, der Mario Rigoni Stern eine unendliche, sich stets erneuernde Bewunderung entgegenbringt, sich wie ein Palimpsest ausnimmt. Der so bezaubernden landschaftlichen Folie, die den Zuschauer durch ihre Farbenpracht und Farbschattierungen, ihre Lichtnuancen und Lichtspiele, ihre optisch erfahrbare Vielfalt dermaßen in ihren Bann zieht, liegt nämlich ein Gewebe zugrunde, eine semantische Vernetzung, sozusagen ein Text, an dem die norditalienischen Soldaten der beiden sich bekämpfenden Lager in den verhängnisvollen Jahren 1915-1918 mitgeschrieben haben. Dieser Text ist allerdings nur noch mit zunehmender

33 Siehe dazu den Katalog dieser Ausstellung, in der die alltäglichen Gegenstände des Ersten Weltkriegs im Mittelpunkt standen: Der Erste Weltkrieg in 100 Objekten.

34 In der Erzählung Ritorno nel Lager I/B kommentiert der Erzähler seine Faszination für die Topographie und für die Kartographie und stellt fest, dass die Besonderheiten der von ihm durchwanderten Landschaften sich in seinem Gedächtnis unauslöschlich eingeprägt haben. Dabei wird das eigene Gedächtnis als ein persönliches Archiv gekennzeichnet: »nunmehr hat sich in meinem Gedächtnis ein Archiv von Bildern gebildet, welche durch Straßen, Schienen, Flüsse, Seen und Städte miteinander verbunden sind « (Ritorno nel Lager I/B, S. 67: »ormai nella memoria si è formato un archivio d'imagini legate da strade, ferrovie, fiumi, laghi e città «).

35 Fantasie di ferragosto, S. 235: »Rivedo in quei nomi, in quelle date tanto della mia vita, della vita del paese, la storia grande e quella piccola. Avevano ragione i greci quando dicevano che le muse sono le figlie della Memoria.« 
Mühe lesbar, da einerseits die Spuren des Ersten Weltkriegs mit der Zeit immer undeutlicher werden, und da andererseits die jüngeren Generationen den Ersten Weltkrieg als ein weit zurückliegendes Ereignis wahrnehmen. Diese Distanz in der Wahrnehmung wird mit dem Verfließen der Zeit zu einer immer größeren Bewusstseinskluft. Der 2008 verstorbene Schriftsteller hätte die Publikationswelle, die im Gedenkjahr 2015 zu beobachten gewesen ist, zweifelsohne begrüßt, denn sie reaktiviert die kollektive Erinnerung an die Geschehnisse im Ersten Weltkrieg. Neben zahlreichen, die Lokalgeschichte betreffenden historischen Studien sind in den vergangenen Monaten recht viele Reise- und Wanderführer für Norditalien erschienen, die die Wanderer und Touristen dazu auffordern wollen, sich die Spuren und Orte des Ersten Weltkriegs im Friaul, im Vicentino, am Isonzo usw. anzuschauen..$^{36}$

Der Palimpsest-Struktur der norditalienischen Berglandschaften begegnen wir in Mario Rigoni Sterns Erzählwerk in verschiedenen Variationen und Ausformungen. So erscheinen uns in der Erzählung I miei sentieri sotto la neve (Meine Pfade unter der Schneedecke) die Berglandschaften am Altipiano di Asiago als eine Überlagerung von mindestens drei verschiedenen Schichten. Unter der winterlichen Schneedecke befindet sich nämlich die Naturlandschaft, welche wiederum die Spuren des Ersten Weltkriegs allmählich überdeckt hat:

Dort ist das Gebirge still und öde. Es betritt jetzt niemand mehr den Maultierpfad, den die Österreicher einst angelegt hatten, um in die Nähe des Berges Ortigara zu gelangen, und wo ich eines Tages die Metallspitze des Bergstocks fand, die hier im Bücherregal liegt. Der Schnee, der in den letzten Tagen mächtig gefallen ist, hat die Pfade der Hirten, die Köhlereien, die Schützengräben des Ersten Weltkriegs, die Abenteuer der Jäger ausgelöscht. Und unter diesem Schnee leben meine Erinnerungen. ${ }^{37}$

Es überlagern sich hier verschiedene Sinnschichten und Geschichtsinhalte, deren potentiell dynamisches Zusammenspiel durch den Erzähler aktiviert werden soll. Bezeichnenderweise - und dies ist bei Rigoni Stern oft der

36 Z.B. Fabi: Andar per trincee sul Carso della Grande Guerra. Es hätte keinen Sinn, hier weitere Beispiele solcher Publikationen mit starkem Lokalbezug aufzulisten. Es sei hier bloß auf das schon zitierte, wissenschaftlich fundierte Buch des Historikers Marco Mondini verwiesen (Andare per i luoghi della Grande Guerra), das einen sehr klaren Überblick über das Kriegsgeschehen in Norditalien und seine Spuren vermittelt, auf diese Weise einen besonders wertvollen Beitrag

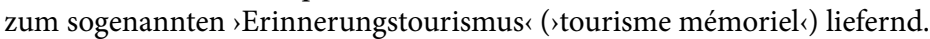

37 I miei sentieri sotto la neve, S. 124: »Lassù la montagna è silenziosa e deserta. Lungo la mulattiera che gli austriaci costruirono per giungere nei pressi dell'Ortigara, dove un giorno raccolsi la punta ferrata del Bergstock che è qui sulla libreria, ora non passa più nessuno. La neve che in questi giorni è caduta abbondante ha cancellato i sentieri dei pastori, le aie dei carbonai, le trincee della Grande Guerra, le avventure dei cacciatori. E sotto quella neve vivono i miei ricordi.« 
Fall - kommt es bei der Beschreibung dieser Palimpsest-Struktur auf die Verbindung von eigenem Schicksal und historischer Bezugnahme, von Geschichten und Geschichte an. In der eben zitierten Erzählung kommt auch die Anekdote von dem wie erlahmt auf einem Stein sitzenden Jüngling vor, dem der Erzähler auf einem seiner Spaziergänge begegnet sei: In ihm habe der Anblick eines in die Dunkelheit getauchten Berges einen derartigen existentiellen Schock ausgelöst, dass er sich kaum mehr zu rühren gewagt habe. Der Jüngling habe nämlich die semantische Dichte dieses Berges konfus wahrgenommen und sei durch diesen erhaben-bedeutungsvollen Anblick in einen wortwörtlich faszinierten Schrecken versetzt worden: »Er saß auf jenem Stein und konnte den Mut nicht dazu aufbringen, sich zu bewegen und dem nächtlichen Berg gegenüberzustehen, der hier so beladen mit Geschichten stand, die zuweilen düster und tragisch waren, Geschichten von Hirten, Jägern, Soldaten, Köhlern, Schmugglern. « ${ }^{38}$ Der Berg ist also geschichten- und geschichtsträchtig, mit Erinnerungen an zahlreiche individuelle Schicksale und historische Begebenheiten beladen.

An vielen anderen Stellen seines Werkes sowie in seinem in Buchform publizierten Gespräch mit Giulio Milani aktualisiert übrigens Mario Rigoni Stern den uralten Topos vom $>$ liber mundi< bzw. vom $>$ liber naturae $\triangleleft,{ }^{39}$ wobei er diesen Topos mit dem geschichtlichen Bewusstsein eines Menschen des 20. Jahrhunderts reinterpretiert, zu dessen biographischem und gedanklichem Horizont die beiden Weltkriege gehören, dessen Weltanschauung also letztendlich mehr von historischen Traumata als von religiösen Deutungsmustern geprägt ist. In dieser modernen Variante des Topos geht es nicht etwa darum, das Buch der Natur als einen Beweis für eine auf dem Prinzip der Theodizee beruhende, in sich geschlossene Weltanschauung zu lesen, sondern eher darum, durch den Anblick einer Berglandschaft, so schön sie auch erscheinen mag, der historischen Risse und Brüche des 20. Jahrhunderts ansichtig und sich ihrer bewusst zu werden. Dass viele von uns nicht (mehr) dazu fähig sind, dem Buch der norditalienischen Berglandschaften diese historische Dichte abzulesen, leuchtet wohl ein, und so sind wir auf die Hilfe eines Begleiters wie Mario Rigoni Stern angewiesen. Mit seinem scharfen Blick, seinem historischen Wissen und seinen eigenen Kriegserlebnissen macht er uns auf die Spuren des Ersten Weltkrieges aufmerksam und erhellt die Zusammenhänge, die semantischen Bezüge, die zwischen notturna così gravida di storie, anche tetre e drammatiche, di pastori, cacciatori, soldati, carbonai, contrabbandieri." 
jenen nunmehr vereinzelten Zeichen und dem Kriegsgeschehen bestehen. Um es mit einer Metapher auszudrücken: Der Blick des Erzählers fungiert hier wie der `Entwickler im Fotolabor, also wie eine chemische Substanz, die das Unsichtbare sichtbar, das Unlesbare lesbar macht. Interessanterweise erfolgt außerdem dieser Prozess des An-den-Tag-Legens von konkreten Spuren und der Herstellung von Sinnbezügen mittels der Artikulation von Schreiben und Gehen.

Die meisten Erzählungen Mario Rigoni Sterns zum Ersten Weltkrieg sind nämlich zugleich Erzählungen von Spaziergängen oder beinhalten zumindest eine Spaziergangsepisode, als ob diese dynamische Beobachtung der Berglandschaften, dieses erzählerische und körperliche Fortschreiten, dieses physische und sprachliche Durchmessen des Raumes, dieses parallel laufende Beschreiben und Beschreiten eine Voraussetzung für die Erschließung und Sichtbarmachung des historischen Subtextes der landschaftlichen, natürlichen Folie wäre. Rigoni Sterns Erzählwerk aktualisiert eine Poetik der Wanderung, die im deutschsprachigen Bereich u.a. an Robert Walsers poetologisch interpretierbaren Text Der Spaziergang anklingt. Der literarische Erinnerungsprozess wird beim italienischen Autor als Wanderung durch das eigene Gedächtnis dargestellt, und komplementär dazu wird das physische Wandern als Voraussetzung, wenn nicht als Auslöser der Erinnerungsdynamik gekennzeichnet. Nicht von ungefähr steht am Anfang von Rigoni Sterns literarischer Laufbahn, gewissermaßen als poetologisches Vorzeichen für sein gesamtes Werk, der Bericht über einen langen Marsch durch winterliche, endlos erscheinende russische Landschaften. Auch in dieser Hinsicht darf man Il sergente nella neve als erzählerische Keimzelle, als Grundmuster von Rigoni Sterns CEuvre ansehen.

In der Erzählung Autunno (Herbst), die man als besonders exemplarisch ansehen darf, ist diese Poetik des Spaziergangs und der gleichzeitigen Offenbarung von historischen Spuren am Werk. Sie befindet sich in der Sammlung Stagioni (Jahreszeiten). Es wird von einer Bergwanderung berichtet, die der Erzähler mit einem Verwandten (einem Onkel oder Großonkel, der Verwandtschaftsgrad ist nicht deutlich zu identifizieren $)^{40}$ unternimmt, welcher am Ersten Weltkrieg teilgenommen hat und gewisse Orte wiedersehen möchte, die sich in seinem Gedächtnis eingeprägt haben und die er seit dem Krieg nie mehr aufgesucht hat. Eingerahmt ist die Beschreibung dieser

40 Im Laufe der Wanderung wird übrigens der konkrete Verwandtschaftsgrad, der die beiden Figuren miteinander verbindet, zugunsten eines höheren, allgemeineren Brüderlichkeitsgefühls aufgehoben (Autunno, S. 116), das sich von den gemeinsamen Kriegserfahrungen und von der Fähigkeit zur Einfühlung in das Schicksal des Anderen nährt. 
Wanderung durch zwei verhältnismäßig lange Heraufbeschwörungen der durch ihre Herrlichkeit bestechenden Berglandschaften Norditaliens: Die Kriegserinnerungen sind auf diese Weise in eine Naturszenerie eingebettet, deren Weite, Erhabenheit und inspirierende Schönheit mutatis mutandis an die Malerei Giovanni Segantinis erinnern. ${ }^{41}$ Diese erzählerische Verschachtelung trägt zur Einordnung der Kriegsgeschehen der Jahre 1915-1918 in den größeren Rahmen der ewigen Natur bei, zur Einordnung der linearhistorischen Zeitlichkeit in den sich immer wiederholenden Kreislauf der Natur. Sie ermöglicht es den beiden Wanderern, eine kontemplative Haltung einzunehmen (mehrmals halten sie inne, um die Landschaft von einem jeweils anderen Blickwinkel zu betrachten und zu bewundern), die die Intensität der aufkommenden Gefühle und Erinnerungen beim Anblick der ehemaligen Kriegsschauplätze dämpft, ohne sie jedoch auszulöschen.

Auch in dieser Erzählung wird das Grundmotiv des Palimpsestes eingesetzt und die Wanderung lässt sich ganz deutlich als die Durchführung eines Leseprozesses identifizieren. Am Anfang der Erzählung steht sogar ein Ausdruck, den man als programmatisch verstehen dürfte: »im Herbst lässt sich der Wald in aller Deutlichkeit lesen ${ }^{42}$ Zwar geht es hier primär um die frischen Spuren der Tiere auf der weichen Erde, um das Reifen der Früchte und um das fallende Laub, um das Wachsen und Sterben der Pflanzenwelt, aber die Formulierung lässt sich problemlos auf die gemeinsame Wanderung der beiden Verwandten übertragen: Neffe und Onkel unternehmen es hier, die Landschaft zu lesen, die den Bergen innewohnende historische Substanz wieder an den Tag treten zu lassen. In der Erzählung wird die Überlagerung von Natur und Geschichte durch ein ständiges Hin und Her zwischen kurzen, aber wunderbar formulierten Naturbeschreibungen einerseits und Hinweisen auf das Kriegsgeschehen und auf die Irrwege der italienischen Truppen andererseits zum Ausdruck gebracht. Die erhabene, geisteserhebende Naturschönheit auf der einen, die zerstörerische Kriegsgewalt auf der anderen Seite werden in einem oxymorischen Ausruf des älteren Wanderers zusammengeführt: »herrlich... schrecklich « (»meraviglioso... orribile«). ${ }^{43}$ Diese Wendung trifft ganz besonders auf den

41 Eine ähnliche Einbettung des Menschen in eine weite, kosmische Szenerie finden wir in den Beschreibungen der slawischen Landschaften, z.B. in der Erzählung Quasi una tregua, S. 65. Die Parallelität zwischen den Erzählungen zum Ersten und jenen zum Zweiten Weltkrieg rührt auch von der ähnlichen Stilisierung der beschriebenen Landschaften her sowie von den Echowirkungen, die der Erzähler zwischen norditalienischen Berglandschaften und slawischen Weiten herstellt. 
Berg Ortigara zu, den die beiden Wanderer am Ende ihrer Wanderung in der Ferne erblicken: »Arrigo richtete sich auf, blickte auf den Ortigara, den Berg der Menschenopferung, und vergoss etwas vom Wein über die kleine Mauer: - Für sie, sagte er. - Für sie, sagte auch ich und wiederholte die Geste. - Und für diejenigen von Albanien und von Russland. « ${ }^{44}$

Diese symbolische Geste, die an antike Trankopfer (>libationes`) erinnert und somit uralte Todesriten wieder gegenwärtig macht, vollführen die beiden Wanderer gemeinsam, auch wenn der jüngere, nämlich der Erzähler, am Ersten Weltkrieg nicht teilgenommen hat. Aber aufgrund der Teilnahme am Zweiten Weltkrieg ist ihm ein am eigenen Körper erlebtes Wissen zuteil geworden, das de facto eine Gemeinsamkeit schafft zwischen den beiden hier dargestellten Schicksalen, die so repräsentativ sind für die traumatische Geschichte des 20. Jahrhunderts. Das Kriegserlebnis des Älteren im Gebirgskrieg 1917 und dasjenige des Jüngeren in Russland 1943 gehören zum selben Erfahrungshorizont, was die Empathie des einen mit dem anderen begründet, ${ }^{45}$ wobei dieses tiefe Einfühlungsvermögen sich weniger durch Worte manifestiert als durch einfache, schlichte Gesten. Diese Idee einer grundlegenden, wenn nicht ontologischen Kontinuität zwischen den verschiedenen Kriegserlebnissen hat Mario Rigoni Stern in seinem Erzählwerk mehrmals thematisiert und ist wohl der Grund, warum bei ihm die Erzählungen zum Ersten Weltkrieg mit den Erzählungen zum Zweiten Weltkrieg in Parallele gebracht werden müssen (und umgekehrt): Die Erzählwerke zu den beiden Weltkriegen erhellen sich sozusagen gegenseitig. Als Pendant zur eben besprochenen Erzählung Autunno steht in Rigoni Sterns Werk der Bericht über die Rückkehr des Schriftsteller-Ich zum Ort der eigenen Kriegsgefangenschaft, Ritorno nel Lager I/B (Rückkehr zum Kriegsgefangenenlager $I / B$ ). Auch hier entfaltet sich die Erzählung als Spaziergang durch eine geschichtsträchtige Landschaft, auch hier alterniert das Staunen angesichts der Schönheit der slawischen Landschaften mit dem schrecklichen Wissen um das dort Geschehene, auch hier wirkt die Diskrepanz zwischen dem landschaftlichen Idyll und der Grausamkeit des Kriegsgeschehens beinahe obszön. Natur und Geschichte überlagern sich in versò un po' di vino oltre il muretto: - Per loro, - disse. - Per loro, - dissi anch'io ripetendo il gesto. - E per quelli dell’Albania e della Russia.«

45 »Einige Jahre zuvor hatte ich dieselben Gefühle an den Ufern des Don empfunden.« (ebd., S. 114: »Qualche anno prima erano stati sentimenti che anch'io avevo provato sulle rive del Don.«); »Was sich für ihn im November 1917 ereignet hatte, hatte sich auch für mich nach dem 8. September 1943 ereignet. « (ebd., S. 123: „Come era stato per lui nel novembre del '17 era stato anche per me dopo l'8 settembre 1943.«); vgl. Miserere nel vento dei Carpazi, S. 13. 
einem sonderbaren, zur Reflexion auffordernden Palimpsest des Grauens, ${ }^{46}$ und nichts anderes als eine sich immer wiederholende kontemplative, zugleich skeptische und hoffungsvolle Meditation über dieses Ineinander von landschaftlicher Herrlichkeit und menschlicher Gewalt, von (hier etymologisch zu verstehendem) >Kosmos` und kriegerischer Sinnlosigkeit bieten Mario Rigoni Sterns Erzählungen.

\section{Literaturverzeichnis}

Der Erste Weltkrieg in 100 Objekten. Hg. von der Stiftung Deutsches Historisches Museum. Berlin: Stiftung DHM/Theiss 2014.

Fabi, Lucio: Andar per trincee sul Carso della Grande Guerra. Trieste: Transalpina 2014. Isnenghi, Mario: Il mito della Grande Guerra. Bologna: Il Mulino ${ }^{72} 214$ ( $\left.{ }^{1} 1989\right)$.

Labanca, Nicola (Hg.): Dizionario storico della Prima Guerra mondiale. Roma, Bari: Laterza 2014.

Labanca, Nicola; Überegger, Oswald (Hgg.): La Grande Guerra italo-austriaca 1914-1918. Bologna: Il Mulino 2014 [deutsche Version: Krieg in den Alpen. Österreich-Ungarn im Ersten Weltkrieg (1914-1918). Wien, Köln, Weimar: Böhlau 2014.

Mondini, Marco: Andare per i luoghi della Grande Guerra. Bologna: Il Mulino 2015.

Rigoni Stern, Mario: Alpini im russischen Schnee [Il sergente nella neve]. Übers. von Hans Hinterhäuser. Heidelberg: Drei Brücken 1954.

Rigoni Stern, Mario: Amore e morte nel bosco incantato. In: ders.: Tra due guerre e altre storie. Torino: Einaudi 2000.

Rigoni Stern, Mario: Autuno. In: ders.: Stagioni. Torino: Einaudi 2006.

Rigoni Stern, Mario: Cartoline. In: ders.: Sentieri sotto la neve. Torino: Einaudi 1998.

Rigoni Stern, Mario: Così a diciottanni andammo in guerra. In: ders.: Tra due guerre e altre storie. Torino: Einaudi 2000.

Rigoni Stern, Mario: Fantasie di Ferragosto. In: ders.: Tra due guerre e altre storie. Torino: Einaudi 2000.

Rigoni Stern, Mario: Füchse unter Sternen [Il bosco degli urogalli]. Übers. von Alice Vollenweider. Zürich: Fretz \& Wasmuth 1964.

Rigoni Stern, Mario: Geblendet und betrogen [Lultima partita a carte]. Übers. von Verena von Koskull. Berlin: Berenberg 2005.

46 In einer makabren Variante des Palimpsest-Motivs werden die Berglandschaften zu einem riesigen Friedhof, zu einem Beinhaus der zahl- und namenlosen Soldaten, die im Gebirgskrieg ihren Untergang fanden - zu einem Beinhaus der Geschichte. So habe sich der Schriftsteller Emilio Lussu geweigert, die Gegend des Altipiano di Asiago wieder zu betreten, und habe diese Weigerung wie folgt begründet: »Mir käme es dann vor, als würde ich über das Fleisch meiner Brüder gehen." (Rigoni Stern/Milani: Storia di Mario, S. 84: »È come se dovessi camminare sulla carne dei miei fratelli.«) Vgl. dazu auch Autunno, S. 117: »Da qui in avanti è come se dovessi camminare tra i morti.« (»Von hier an ist es, als würdest du unter Toten wandeln.«) Die Bergwanderung wird auf einmal zur Höllenfahrt, zum (pietät- und ehrfurchtsvollen) Betreten des Totenreiches. 
Rigoni Stern, Mario: I racconti di guerra. Introduzione di Folco Portinari. Torino: Einaudi 2006.

Rigoni Stern, Mario: I miei sentieri sotto la neve. In: ders.: Sentieri sotto la neve. Torino: Einaudi 1998.

Rigoni Stern, Mario: Il naufragio dei contadini. In: ders.: I racconti di guerra. Introduzione di Folco Portinari. Torino: Einaudi 2006.

Rigoni Stern, Mario: Il Piave mormorava. In: ders.: I racconti di guerra. Introduzione di Folco Portinari. Torino: Einaudi 2006.

Rigoni Stern, Mario: La ricostruzione dell'Altipiano di Asiago. In: ders.: I racconti di guerra. Introduzione di Folco Portinari. Torino: Einaudi 2006.

Rigoni Stern, Mario: Malghe. In: ders.: Tra due guerre e altre storie. Torino: Einaudi 2000. Rigoni Stern, Mario: Miserere nel vento dei Carpazi. In: ders.: I racconti di guerra. Introduzione di Folco Portinari. Torino: Einaudi 2006.

Rigoni Stern, Mario: Musil in trincea. In: ders.: I racconti di guerra. Introduzione di Folco Portinari. Torino: Einaudi 2006.

Rigoni Stern, Mario: Osteria di confine. In: ders.: Sentieri sotto la neve. Torino: Einaudi 1998. Rigoni Stern, Mario: Quasi una tregua. In: ders.: Aspettando l'alba. Torino: Einaudi 2004. Rigoni Stern, Mario: Ritorno nel Lager I/B. In: ders.: Aspettando l'alba. Torino: Einaudi 2004. Rigoni Stern, Mario: Scampanio profano. In: ders.: Amore di confine. Torino: Einaudi 1986. Rigoni Stern, Mario: Segni lontani. In: ders.: Sentieri sotto la neve. Torino: Einaudi 1998. Rigoni Stern, Mario: Tönle [Storia di Tönle]. Übers. von Gerda Lederer. Stuttgart: ComMedia \& arte 1988.

Rigoni Stern, Mario; Milani, Giulio: Storia di Mario. Mario Rigoni Stern e il suo mondo. Conversazione a cura di Giulio Milani. Massa: Transeuropa 2008. 